\title{
PARASITISM OF GUM LEAF SKELETONISER (URABA LUGENS) IN NEW ZEALAND
}

\author{
S. MANSFIELD, D.J. KRITICOS, K.J.B. POTTER and M.C. WATSON \\ Forest Research, PB 3020, Rotorua, New Zealand
}

Corresponding author: sarah.mansfield@ensisjv.com

\begin{abstract}
The gum leaf skeletoniser (Uraba lugens), a significant pest in Australia, is now well established on Eucalyptus spp. in the Auckland region. One larval parasitoid (Meteorus pulchricornis) and two pupal parasitoids (Xanthopimpla rhopaloceros and Anacis sp.) were recorded from $U$. lugens collected in southwest Auckland. Parasitism of $M$. pulchricornis and X. rhopaloceros against $U$. lugens and other hosts in New Zealand (Helicoverpa armigera and Epiphyas postvittana, respectively) was compared using no-choice and choice tests under controlled conditions. Uraba lugens is a suitable host for development of both $M$. pulchricornis and X. rhopaloceros. Choice tests revealed that $M$. pulchricornis prefers $H$. armigera larvae to $U$. lugens. Attack by $X$. rhopaloceros occurred only when host pupae were presented within their cocoons. Meteorus pulchricornis may compete with proposed classical biological control agents introduced against $U$. lugens, while $X$. rhopaloceros is more likely to complement them.

Keywords: biological control, Eucalyptus, Nolidae, parasitoid, Uraba lugens.
\end{abstract}

\section{INTRODUCTION}

The gum leaf skeletoniser, Uraba lugens (Walker) (Lepidoptera: Nolidae), occurs in every state of Australia except the Northern Territory and has 1-2 generations per year with 6-14 larval instars (Allen \& Keller 1991; Elliott et al. 1998). Larvae feed on a wide range of Eucalyptus spp. and closely related species such as Angophora and Lophostemon (Morgan \& Cobbinah 1977; Farr 2002). Uraba lugens became the focus of a long-term management programme after the discovery of a widespread infestation in southwest Auckland, particularly suburbs surrounding Auckland International Airport, during 2001 (Ross 2003). The Auckland populations have two generations each year, with larvae present from approximately January to March (summer generation) and May to October (winter generation, Withers et al. 2003). Selection and host specificity testing of potential biological control agents for $U$. lugens currently form a significant part of this management programme.

In Australia $U$. lugens supports an extensive parasitoid fauna; some are polyphagous, others are thought to be host specific (Austin \& Allen 1989). Three larval parasitoids and one larval-prepupal parasitoid have been selected for importation into containment at Forest Research. These are Cotesia urabae Austin and Allen, Dolichogenidea eucalypti Austin and Allen (Hymenoptera: Braconidae), Eriborus sp. (Hymenoptera: Ichneumonidae) and Euplectrus sp. (Hymenoptera: Eulophidae) (Mansfield 2005). In New Zealand, numerous endemic and exotic lepidopteran parasitoids are present (Berry 2005), some of which may be able to attack $U$. lugens. Such parasitoids would be potential competitors of introduced biological control agents specific to $U$. lugens.

The aim of this study was to identify and record what parasitoids (if any) were already attacking $U$. lugens populations in southwest Auckland and to qualitatively gauge their likely impact upon these populations. If parasitism is substantial and sustained, then the need for additional control agents would be questionable, and the presence of local 
parasitoids might create difficulties in getting new biological control agents established. When parasitoids were identified in the Auckland populations of $U$. lugens, their attack behaviour on $U$. lugens and a known host were compared using no-choice tests under controlled conditions. Choice tests were also conducted to assess the parasitoids' preference for the novel host compared with the known host.

\section{Field collection}

METHODS

Collections were made from infested trees in southwest Auckland at approximately weekly intervals for the summer generation and fortnightly for the winter generation ( $\geq 10$ U. lugens per collection). Twenty-two larvae and 31 pupae were collected from 21 February to 26 March 2004. This period corresponds to the late instar larval and pupal stages of the summer generation (Withers et al. 2003). One hundred and seventy-five larvae and 130 pupae were collected from 2 August to 22 November 2004. This period corresponds to the middle instar larval and pupal stages of the winter generation. Insects were sent to Forest Research, Rotorua, by overnight courier where they were reared in containment at $22^{\circ} \mathrm{C}, 70 \% \mathrm{RH}, 12: 12 \mathrm{~h}$ light:dark. Larvae from each collection were fed on Eucalyptus nitens foliage in plastic pottles $(395 \mathrm{ml}$, with small air holes punched into lid) until pupation or emergence of a parasitoid cocoon. Uraba lugens and parasitoid pupae were stored in individual plastic cups $(30 \mathrm{ml})$ until adults emerged. Parasitoid specimens were identified by a specialist taxonomist (J.A. Berry, Landcare Research). Insect cultures

All insects were kept in the laboratory at $22^{\circ} \mathrm{C}, 70 \% \mathrm{RH}, 12: 12 \mathrm{~h}$ light:dark. Uraba lugens were reared in a perspex cage $(1.0 \mathrm{~m} \times 0.7 \mathrm{~m} \times 0.7 \mathrm{~m})$ with screen windows $(200 \mathrm{~mm} \times 300 \mathrm{~mm})$ on three sides and two mesh sleeves $(20 \mathrm{~mm}$ diameter $)$ at the front. Larvae were fed cut stems of $E$. nitens foliage that were put into water-filled containers. Fresh foliage was collected weekly from a local plantation of $E$. nitens and kept in water at $4^{\circ} \mathrm{C}$ until needed.

At regular intervals, HortResearch (Mt. Albert, Auckland) supplied the parasitoid hosts, Helicoverpa armigera (Hübner) (Lepidoptera: Noctuidae) eggs and Epiphyas postvittana (Walker) (Lepidoptera: Tortricidae) larvae, each with appropriate artificial diet for use in experimental trials. Approximately 70 adult Meteorus pulchricornis (Wesmael) (Hymenoptera: Braconidae) were supplied by HortResearch to start a culture of this parasitoid on $H$. armigera. The parasitoids were kept in a perspex cage $(0.8 \mathrm{~m}$ x $0.7 \mathrm{~m}$ x $0.5 \mathrm{~m}$, otherwise similar to $U$. lugens cage) with honey streaked on the walls and ceiling as a food source. Approximately 30-50 H. armigera larvae were exposed to the parasitoids every 2-3 days. Exposed larvae were kept in individual plastic cups $(30 \mathrm{ml})$ and supplied with artificial diet until a parasitoid cocoon appeared or the larva pupated. Parasitoid cocoons were collected every 2-3 days and held in Petri dishes until parasitoids emerged.

A culture of Xanthopimpla rhopaloceros (Krieger) (Hymenoptera: Ichneumonidae) was developed from parasitoids that emerged out of field-collected $U$. lugens pupae. Parasitoids were kept in a plastic Bugdorm (300 mm x $300 \mathrm{~mm}$ x $300 \mathrm{~mm}$, Bioquip) with honey streaked on the walls and ceiling. Epiphyas postvittana proved to be unsuitable as a rearing host for this parasitoid (see results and discussion). Instead up to $30 \mathrm{U}$. lugens pupae were exposed to the parasitoids every 2-4 days, depending on availability. After exposure, pupae were held in plastic pottles $(535 \mathrm{ml})$ until moths or parasitoids emerged.

\section{No-choice and choice tests}

Female M. pulchricornis were fed honey and kept without access to hosts for 3-4 days after emergence. Each female was used only once. The experimental arena for nochoice and choice tests was a glass Petri dish (100 mm diameter). For the no-choice tests, 10 larvae were added to the Petri dish also containing either one E. nitens leaf (U. lugens) or one piece of diet $(H$. armigera). Three size classes of larvae were tested for each host species with mean head capsule widths of $0.2,0.4$ and $0.8 \mathrm{~mm}$. These size classes 
corresponded to $2 \mathrm{nd}$, 3rd and 4th instar U. lugens or 1st, 2nd and 3rd instar H. armigera. Honey was streaked on the lid of the Petri dish. One female parasitoid was released in each dish for $24 \mathrm{~h}$. After the exposure period, the larvae were removed and kept with appropriate food until pupation or a parasitoid cocoon appeared. Twenty parasitoids were tested for each host size class, i.e. 120 parasitoids in total. Choice tests using 3rd instar $U$. lugens and 2 nd instar $H$. armigera ( $\mathrm{n}=20$ parasitoids), were conducted in a similar fashion except each parasitoid was offered five $U$. lugens and five $H$. armigera larvae simultaneously. No-choice tests were analysed by two-way ANOVA and choice tests by a paired sample t-test (SAS version 8.2).

As only a limited number of $U$. lugens pupae were available to rear $X$. rhopaloceros, too few parasitoids were reared to standardise parasitoid age across the no-choice and choice experiments. However, all female parasitoids were given a minimum of $48 \mathrm{~h}$ with honey and males present but without access to hosts before testing and females were used only once. A plastic pottle $(535 \mathrm{ml})$ was used as the experimental arena for $X$. rhopaloceros due to its large body size and need for more space to successfully attack hosts. Each female was offered either five U. lugens or E. postvittana pupae for $24 \mathrm{~h}$ $(\mathrm{n}=10$ parasitoids for each host) during the no-choice tests. Choice tests were performed under similar conditions with each parasitoid female offered five U. lugens and five E. postvittana pupae simultaneously ( $\mathrm{n}=10$ parasitoids). Host pupae were held at $13^{\circ} \mathrm{C}$ for no more than 7 days to slow pupal development until sufficient pupae were available to conduct a test.

\section{Field collection}

\section{RESULTS AND DISCUSSION}

One larval and two pupal parasitoids were reared from field-collected $U$. lugens (Table 1). Larval parasitism appeared similar for summer and winter generations of $U$. lugens whereas pupal parasitism declined in winter. However, the small sample size of the summer collection means such comparisons cannot be made with confidence.

Meteorus pulchricornis is a solitary endoparasitoid with a cosmopolitan distribution that attacks larvae from eight lepidopteran families (Huddleston 1980; Fuester et al. 1993). Since this parasitoid was first recorded in New Zealand during 1996 it has been reared from four different species, two tortricids, one noctuid and one exotic lymantriid (Berry 1997). Meteorus pulchricornis has also been recorded from U. lugens in Australia (J.G. Charles, pers. comm.). The records reported here are likely to be an underestimate of the impact of this species on U. lugens in Auckland because more recent larval collections from the summer generation of 2004-05 indicate parasitism by M. pulchricornis has increased to approximately $20 \%$ (A.M. Barrington, pers. comm.).

Xanthopimpla rhopaloceros is a solitary pupal parasitoid that was introduced to New Zealand from Australia for the control of light brown apple moth and is now widespread (Thomas 1989). This insect has been recorded as an occasional parasitoid of $U$. lugens in Australia (Allen 1990a). The second pupal parasitoid was identified as Anacis sp. (Hymenoptera: Ichneumonidae) and is the only species in this genus in New Zealand (J.A. Berry, pers. comm.). There are more than 50 undescribed species within this genus in Australia (Gauld 1984), but there are no known records of this parasitoid from U. lugens in its native range. A laboratory culture of this species could not be established so no further tests of its attack behaviour were possible. 
TABLE 1. Number of Uraba lugens larvae and pupae collected, percentage parasitism, and number of each parasitoid species emerged. Field collections were made in southwest Auckland from February to November 2004.

\begin{tabular}{lcccccc}
\hline \multicolumn{2}{c}{ Uraba lugens } & Generation & Number Parasitism & Meteorus & Xanthopimpla & Anacis \\
collected & $(\%)$ & pulchricornis & rhopaloceros & sp. \\
\hline Larvae & Summer & 22 & 4.5 & 1 & & \\
\multirow{5}{*}{ Pupae } & Winter & 175 & 4.5 & 8 & 5 & 1 \\
& Summer & 31 & 19.3 & & 2 & 9 \\
\hline
\end{tabular}

No-choice and choice tests

All instars of each host species tested were parasitised by M. pulchricornis in the nochoice tests although significantly more $H$. armigera were parasitised than $U$. lugens $(\mathrm{P}<0.0001$, Table 2). Parasitism was more successful on some instars than others in terms of percentage parasitism and the number of parasitoid larvae per female $(\mathrm{P}<0.0001)$, with M. pulchricornis most successful on 2 nd instar H. armigera. The 2 nd and $3 \mathrm{rd}$ instars of $U$. lugens were more heavily parasitised than the 4th instar larvae. Development time (days to parasitoid pupation) was shorter on H. armigera than U. lugens $(\mathrm{P}<0.0001)$ and was also shorter on the oldest instar tested for each species $(\mathrm{P}<0.0001)$. Adult parasitoid emergence was high for both host species, ranging from $94-100 \%$ of parasitoid pupae. In the choice tests, although the parasitism of $H$. armigera declined compared with the no-choice tests, $M$. pulchricornis still parasitised twice as many H. armigera as $U$. lugens $(\mathrm{P}=0.002$, Table 3$)$. Adult emergence remained high for $H$. armigera but declined for $U$. lugens compared with the no-choice tests.

TABLE 2. Parasitism and adult emergence under no-choice conditions of two host species by Meteorus pulchricornis $(n=20$ females for each host instar), with number of parasitoid larvae (mean $\pm \mathrm{SE}$ ) per female and development time (days to parasitoid pupation, mean $\pm \mathrm{SE}$ ).

\begin{tabular}{lccccc}
\hline $\begin{array}{l}\text { Host/ } \\
\text { Instar }\end{array}$ & No. of hosts & $\begin{array}{c}\text { Parasitism } \\
(\%)\end{array}$ & $\begin{array}{c}\text { Emergence } \\
(\%)\end{array}$ & $\begin{array}{c}\text { Larvae per } \\
\text { female }\end{array}$ & Development time \\
\hline H. armigera & & & & & \\
1st & 194 & 4.1 & 100 & $0.4 \pm 0.18$ & $14.00 \pm 0.46$ \\
2nd & $166^{1}$ & 94.6 & 95.5 & $7.85 \pm 0.44$ & $15.09 \pm 0.18$ \\
3rd & 196 & 40.8 & 93.8 & $4.0 \pm 0.59$ & $10.23 \pm 0.26$ \\
U. lugens & & & & & \\
2nd & 200 & 16 & 100 & $1.6 \pm 0.31$ & $28.71 \pm 0.68$ \\
3rd & 199 & 17.6 & 97.1 & $1.75 \pm 0.32$ & $27.34 \pm 0.67$ \\
4th & 200 & 9.5 & 94.7 & $0.95 \pm 0.23$ & $19.74 \pm 0.51$ \\
\hline
\end{tabular}

${ }^{1}$ Some host larvae lost through cannibalism.

TABLE 3. Parasitism and adult emergence under choice conditions of two host species by Meteorus pulchricornis ( $\mathrm{n}=\mathbf{2 0}$ females for each host) and the number of parasitoid larvae (mean $\pm \mathrm{SE}$ ) produced per female.

\begin{tabular}{lccccc}
\hline Host species & Instar & No. of hosts & Parasitism (\%) & Emergence (\%) & Larvae per female \\
\hline H. armigera & 2nd & 97 & 43.3 & 100 & $2.1 \pm 0.30$ \\
U. lugens & 3rd & 89 & 21.3 & 57.9 & $0.8 \pm 0.23$ \\
\hline
\end{tabular}

Fuester et al (1993) reported attack by M. pulchricornis on 1st to 3rd instar larvae of the gypsy moth, Lymantria dispar (L.) (Lepidoptera: Lymantriidae), with the highest 
parasitism occurring on the 2 nd instar. Parasitoids took from $10-22$ days at $24^{\circ} \mathrm{C}$ to reach pupation in this host, while Askari et al. (1977) found M. pulchricornis reached pupation after 11 days at $25^{\circ} \mathrm{C}$ in larvae of the greater wax moth, Galleria mellonella (L.) (Lepidoptera: Pyralidae). These results appear comparable to H. armigera in this study, despite the different temperature conditions.

No $X$. rhopaloceros emerged from E. postvittana pupae that were exposed in the no-choice tests and only one male parasitoid emerged from this host in the choice tests. Attempts to establish a culture of this parasitoid on E. postvittana were also unsuccessful. While this was unexpected, further observation suggested that the female parasitoids frequently failed to recognise host pupae that were not enclosed within their original cocoon. The E. postvittana larvae used in this trial were reared on artificial diet in small tubes and did not spin a complete cocoon at pupation under these conditions, even when apple foliage was supplied as a potential substrate. Also, the bare pupae were able to evade approaching parasitoids by wriggling across the floor of the arena.

The importance of the cocoon for host recognition was further demonstrated when the response of female parasitoids to $U$. lugens pupae with and without their cocoons was observed. Only those pupae still in their cocoons were attacked. Following these observations, only $U$. lugens pupae that were fully enclosed in their cocoons were used for experiments and culture maintenance. A total of 22 parasitoids (10 females, 12 males) emerged from 50 U. lugens pupae exposed in the no-choice tests (this equates to $44 \%$ parasitism). Mean $( \pm$ SE) development time was $28( \pm 2)$ days and ranged from 16-41 days over the duration of the tests. Since there were insufficient pupae to standardise host age, some of this variability may be linked to variation in host development at the time of attack. No parasitoids emerged from U. lugens pupae exposed during the choice tests, making it impossible to assess the preference of $X$. rhopaloceros for these two hosts. It is not clear what caused this failure of attack although the greater age of the female parasitoids used in the choice tests compared with the no-choice tests may have been a contributing factor.

\section{Interaction with proposed biological control agents}

Uraba lugens was more heavily parasitised by M. pulchricornis and X. rhopaloceros under controlled conditions than under natural conditions, suggesting either these parasitoids have yet to achieve their full impact against $U$. lugens or another factor is reducing their efficiency in the field, e.g. the presence of alternative host species. Eleven primary parasitoids have been identified from U. lugens in Australia (Allen 1990a), so it is likely that the New Zealand population could support more parasitoid species than are currently present.

The pupal parasitoids X. rhopaloceros and Anacis sp. are unlikely to interfere directly with any of the possible host-specific control agents introduced against $U$. lugens. However, M. pulchricornis attacks some or all of the larval instars targeted by the candidate control agents. Therefore this parasitoid is likely to hinder the initial survival of host-specific parasitoids through direct competition for hosts and perhaps superparasitism. Once a host-specific control agent is well established, it may have a competitive advantage over M. pulchricornis. For example, C. urabae and D. eucalypti actively move through gregarious patches of $U$. lugens larvae, attacking as they go (Allen 1990b). Experimental studies of the interactions between M. pulchricornis and the candidate agents in containment would help predict the impact of inter-specific competition, if host-specific agents are to be released in future.

\section{CONCLUSIONS}

In New Zealand, one larval and two pupal parasitoids have so far been reared from $U$. lugens field populations. Uraba lugens is a suitable host for M. pulchricornis and $X$. rhopaloceros although $H$. armigera is a more preferred host than $U$. lugens for M. pulchricornis. The life histories of the two pupal parasitoids will avoid any direct competition between these species and the candidate biological control agents. In contrast, the larval parasitoid M. pulchricornis can be expected to compete with all four candidates. The outcome of such competition is unknown, although the specialised attack behaviour shown by $C$. urabae and $D$. eucalypti could give them an advantage. 


\section{ACKNOWLEDGEMENTS}

The assistance of Chris Scott, Chris Inglis and Les Renney (Vigil) with field collections is appreciated. Thank you also to Anne Barrington, Asha Chhagan and John Charles (HortResearch) for providing insects. This project was funded by MAF Biosecurity.

\section{REFERENCES}

Allen GR 1990a. Uraba lugens Walker (Lepidoptera: Noctuidae): larval survival and parasitoid biology in the field in South Australia. Journal of the Australian Entomological Society 29: 301-312.

Allen GR 1990b. Influence of host behavior and host size on the success of oviposition of Cotesia urabae and Dolichogenidae eucalypti (Hymenoptera: Braconidae). Journal of Insect Behavior 3(6): 733-749.

Allen GR, Keller MA 1991. Uraba lugens (Lepidoptera: Noctuidae) and its parasitoids (Hymenoptera: Braconidae): temperature, host size, and development. Environmental Entomology 20(2): 458-469.

Askari A, Mertins JW, Coppel HC 1977. Developmental biology and immature stages of Meteorus pulchricornis in the laboratory. Annals of the Entomological Society of America 70(5): 655-659.

Austin AD, Allen GR 1989. Parasitoids of Uraba lugens Walker (Lepidoptera: Noctuidae) in South Australia, with description of two new species of Braconidae. Transactions of the Royal Society of South Australia 113(4): 169-184.

Berry JA 1997. Meteorus pulchricornis (Wesmael) (Hymenoptera: Braconidae: Euphorinae), a new record for New Zealand. New Zealand Entomologist 20: 4548.

Berry JA 2005. Checklist of New Zealand Hymenoptera. http://www.landcareresearch. co.nz/research/biodiversity/invertebratesprog/hymenoptera/checklist.asp (Accessed 13 May 2005).

Elliott HJ, Ohmart CP, Wylie FR 1998. Insect pests of Australian forests: ecology and management. Inkata Press, Melbourne. 214 p.

Farr JD 2002. Biology of the gum leaf skeletoniser, Uraba lugens Walker (Lepidoptera: Noctuidae), in the southern jarrah forest of Western Australia. Australian Journal of Entomology 41: 60-69.

Fuester RW, Taylor PB, Peng H, Swan K 1993. Laboratory biology of a uniparental strain of Meteorus pulchricornis (Hymenoptera: Braconidae), an exotic larval parasite of the gypsy moth (Lepidoptera: Lymantriidae). Annals of the Entomological Society of America 86(3): 298-304.

Gauld ID 1984. An Introduction to the Ichneumonidae of Australia. British Museum (Natural History), London. 413 p.

Huddleston T 1980. A revision of the western Palaearctic species of the genus Meteorus (Hymenoptera: Braconidae). Bulletin of the British Museum, Entomology Series 4(1). $58 \mathrm{p}$.

Mansfield S 2005. Biological control of Uraba lugens - progress report. Forest Health News 150: 1 .

Morgan FD, Cobbinah JR 1977. Oviposition and establishment of Uraba lugens (Walker), the gum leaf skeletoniser. Australian Forestry 40(1): 44-55.

Ross M 2003. Gum leaf skeletoniser long-term management approved. Biosecurity Issue 47: 8 .

Thomas WP 1989. Epiphyas postvittana (Walker), lightbrown apple moth (Lepidoptera: Tortricidae). In: Cameron PJ, Hill RL, Bain J, Thomas WP ed. A review of biological control of invertebrate pests and weeds in New Zealand 1874 to 1987. Technical Communication No. 10. CABI and DSIR, Oxon. Pp. 187-195.

Withers TM, Richardson B, Kimberley M, Kriticos DJ 2003. Modelling the phenology of Uraba lugens (Nolidae). Unpublished report to Ministry of Agriculture and Forestry Biosecurity Authority. 